\title{
Differences in socio-demographic and risk factor profile, clinical presentation, and outcomes between patients with and without RHD heart failure in Sub-Saharan Africa: results from the THESUS-HF registry
}

\author{
Clovis Nkoke ${ }^{1,2}$, Albertino Damasceno ${ }^{3}$, Christopher Edwards ${ }^{4}$, Beth Davison ${ }^{4,5}$, Gad Cotter $^{4,5}$, \\ Mahmoud Sani $^{6}$, Lauren Gaeta ${ }^{4}$, Okechukwu S. Ogah ${ }^{7}$, Charles Mondo ${ }^{8}$, Dike Ojji ${ }^{9}$, Ahmed Suliman ${ }^{10}$, \\ Gerald Yonga $^{11}$, Serigne Abdou $\mathrm{Ba}^{12}$, Anastase Dzudie ${ }^{2,13}$, Karen Sliwa ${ }^{14}$ \\ ${ }^{1}$ Department of Internal Medicine, Buea Regional Hospital, Buea, Cameroon; ${ }^{2}$ Clinical Research Education, Networking and Consultancy, Douala, \\ Cameroon; ${ }^{3}$ Eduardo Mondlane University, Maputo, Mozambique; ${ }^{4}$ Momentum Research Inc., Durham, North Carolina, USA; ${ }^{5} 942$ Inserm- \\ MASCOT, Paris, France; ${ }^{6}$ Department of Medicine, Bayero University Kano/Aminu Kano Teaching Hospital, Kano, Nigeria; ${ }^{7}$ Cardiology Unit, \\ Department of Medicine, University of Ibadan/University College Hospital, Ibadan, Oyo State; ${ }^{8}$ Kiruddu National Referral Hospital, Kampala, \\ Uganda; ${ }^{9}$ Cardiology Unit, Department of Medicine, University of Abuja Teaching Hospital, Abuja, Nigeria; ${ }^{10}$ Faculty of Medicine, University \\ of Khartoum, Khartoum, Sudan; ${ }^{11}$ Department of Medicine, Aga Khan University, Nairobi, Kenya; ${ }^{12}$ Service de cardiologie, Faculte de medecine \\ de Dakar, Dakar, Senegal; ${ }^{13}$ Faculty of Medicine and Biomedical Sciences, University of Yaounde 1, Yaoundé, Cameroon; ${ }^{14}$ Hatter Institute for \\ Cardiovascular Research in Africa, Cape Town, South Africa \\ Contributions: (I) Conception and design: C Nkoke, A Damasceno, B Davison, G Cotter; (II) Administrative support: A Damasceno, B Davison, G \\ Cotter, M Sani, L Gaeta, OS Ogah, G Yonga, A Dzudie, K Silwa; (III) Provision of study materials or patients: A Damasceno, M Sani, OS Ogah, C \\ Mondo, D Ojji, A Suliman, G Yonga, SA Ba, A Dzudie, K Silwa; (IV) Collection and assembly of data: A Damasceno, C Edwards, B Davison, M Sani, \\ OS Ogah, C Mondo, D Ojji, A Suliman, G Yonga, SA Ba, A Dzudie, K Silwa; (V) Data analysis and interpretation: C Nkoke, C Edwards, B Davison; \\ (VI) Manuscript writing: All authors; (VII) Final approval of manuscript: All authors. \\ Correspondence to: Clovis Nkoke. Buea Regional Hospital, Buea, Cameroon. Email: cnkoke@yahoo.com.
}

Background: Rheumatic heart disease (RHD) was found in the THESUS-HF registry to be the third most common cause of acute heart failure (AHF) in Sub-Saharan Africa.

Methods: One thousand six patients with AHF from 9 Sub-Saharan African countries were recruited in THESUS-HF, of which 143 (14.3\%) had RHD-AHF. Clinical characteristics and outcomes in patients with RHD-AHF and non-RHD-AHF were compared. Kaplan-Meier plots for time to all-cause death and/or HF readmission according to the presence of RHD-AHF and non-RHD-AHF were performed and survival distributions compared using the log-rank test. Cox regression was used to determine the hazard ratio of death to day 180 and death or readmission to day 60 after adjusting for confounders.

Results: Patients with RHD-AHF were younger, more often females, had higher rates of atrial fibrillation, had less hypertension, hyperlipidemia and diabetes, had lower BP, and higher pulse rate and better kidney function and echocardiographic higher ejection fraction larger left atria and more diastolic dysfunction. Patients with RHD-AHF had a numerically longer mean stay in the hospital (10.5 vs. 8.8 days) and significantly higher initial hospitalization mortality (9.1\% vs. $3.4 \%$ ).

Conclusions: In conclusion, patients with HF related to RHD were younger, have higher rate of atrial fibrillation and have a worse short-term outcome compared to HF related to other etiologies in Sub-Saharan Africa.

Keywords: Heart failure (HF); rheumatic heart disease (RHD); Africa; outcome

Submitted Feb 24, 2021. Accepted for publication Jun 20, 2021.

doi: $10.21037 / \mathrm{cdt}-21-112$

View this article at: https://dx.doi.org/10.21037/cdt-21-112 


\section{Introduction}

Heart failure (HF) is a major cause of morbidity and mortality in developed and developing countries. Rheumatic heart disease (RHD) is the third leading cause of HF in Africa after hypertension and dilated cardiomyopathies as reported in a recent systematic review and meta-analysis (1). It was also the third most common HF etiology in the multinational sub-Saharan Africa Survey of Heart Failure (THESUS-HF) study (2). RHD diagnosis in Sub-Saharan Africa (SSA) is usually made at an advanced stage of the disease, when severe valve lesions become symptomatic, and there is need for invasive intervention (3). Severe RHD is lethal in the absence of invasive treatment. However, interventions such as percutaneous mitral dilatation, closed heart mitral commissurotomy and open-heart surgery can be performed at only a few centers in Africa and are not usually affordable (4). Given the high prevalence of RHF and the high incidence of RHD-AHF in Africa, the current analysis was performed to compare the socio-demographic, clinical characteristics and outcome between patients admitted with acute heart failure (AHF) due to RHD and non-RHD (RHD-AHF and non-RHD-AHF) and followed during 6 months using data from the multinational THESUS-HF study. We present the protocol in accordance with the STROBE reporting checklist (available at https:// dx.doi.org/10.21037/cdt-21-112).

\section{Methods}

THESUS-HF was a prospective, multicenter, observational study of patients admitted between July 2007 and June 2010 for AHF in 12 hospitals from nine countries in southern, eastern, central, and western sub-Saharan Africa, mostly in Nigeria, Uganda, and South Africa. Eligibility criteria included dyspnoea as the main complaint leading to admission and symptoms and signs of congestive heart failure including rales, oedema, and raised jugular venous pulse. Echocardiographic findings supported the diagnosis which a cardiologist confirmed. Patients' written informed consent was required for entry into the study. Each participating institution's ethics committee approved the study, which conformed to the principles of the Declaration of Helsinki.. Investigators recorded data onto standardized case report forms, and data collected included medical history, medication use, laboratory values, and physical examination with heart failure signs and symptoms. Echocardiographic assessments were made locally and electrocardiograms were read centrally. Tests for human immunodeficiency virus were done if clinically indicated. The occurrence of readmissions and/or death over 6 months was assessed either at clinic visits or through telephone contacts.

\section{Statistical analysis}

Nine patients where the diagnosis of RHD was not available were excluded from all analyses. Results are presented as counts and percentages for categorical variables and means, standard deviation (SD), median and 25 th- 75 th percentiles for continuous variables. Comparison between participants with RHD-HF and those with non RHDHF for demographics, baseline clinical characteristics, medical history, echocardiographic parameters, and baseline medication use were tested using chi square tests for qualitative variables, Wilcoxon rank-sum for ordinal variables, and $t$-test for quantitative variables.

The associations between RHD-AHF or non-RHDAHF clinical outcomes were examined. A $t$-test was used for comparing length of initial hospital stay between groups. In-hospital mortality was compared between groups using a logistic regression model with the odds ratio, 95\% confidence interval, and Wald chi-square $\mathrm{P}$ value presented. For time-to-event outcomes, Kaplan-Meier estimates are presented with the log rank test used for comparison between groups; hazard ratios and $95 \%$ confidence intervals from Cox regression models are also given. Hazard ratios adjusting for predictors found to be associated with readmission or death to day 60 and all-cause death to day 180 are also presented (5). These predictors include history of hyperlipidemia, malignancy, and cor pulmonale and; baseline rales, ejection fraction, BUN, and systolic BP for death or readmission to Day 60 and history of malignancy, cor pulmonale, and HIV; gender, smoking status, and baseline values for systolic BP, creatinine, hemoglobin, heart rate, orthopnea, rales, and edema for death to day 180. Multiple imputation methods were used to account for missing predictors in the adjusted analyses. Rubin's algorithm (SAS PROC MIANALYZE) was used to average parameter estimates across seven imputation datasets. Plots of Kaplan-Meier curves for time to all-cause death and/or HF readmission according to the presence of RHD-HF and non-RHD-HF are shown. Data were analyzed using SAS software version 9.4 (SAS Institute, Cary, NC, USA). A P value $<0.05$ was considered statistically significant. 


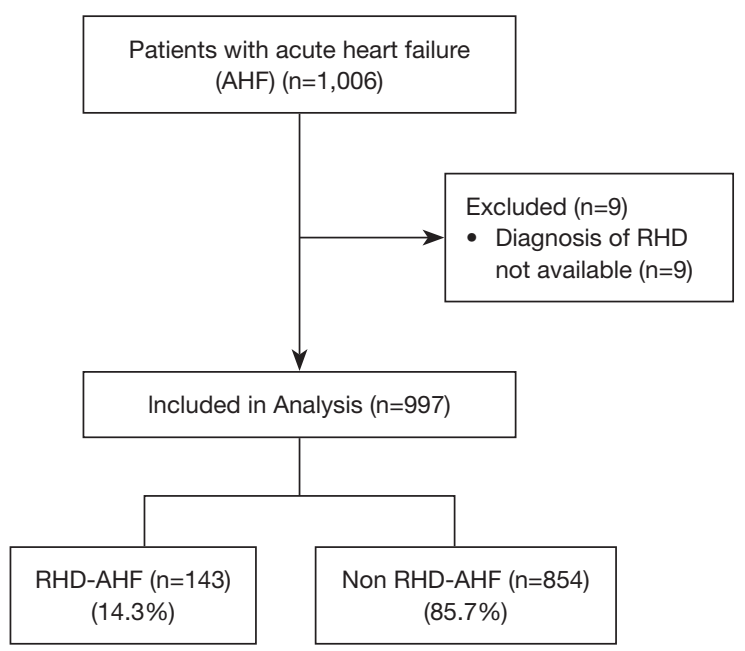

Figure 1 Analysis flow chart.

\section{Results}

\section{Socio-demographic and risk factor profile}

One thousand six $(1,006)$ patients with AHF from nine sub-Saharan African countries were recruited in THESUSHF, of which 997 were included in this analysis. There were 143 patients (14.3\%) with RHD-AHF (Figure 1). Comparison of socio-demographic and risk factor profile is shown in Table 1. Most of the patients were black Africans (99\%). The mean age (SD) was 52.3 (18.3) years, and 489 (49.1\%) were males. Patients with heart failure associated with RHD were younger (42.5 vs. 54.0 years), were more likely to be females, and were more likely to have atrial fibrillation (42.9\% vs. 14.5\%). Patients with non-RHDAHF had higher rates of hypertension, diabetes mellitus, and hypercholesterolemia. In laboratory examinations they had worst kidney function and higher glucose, but less leukocytosis.

\section{Comparison of clinical and echocardiographic characteristics}

Comparison of clinical presentation is shown in Table 2 . Patients with heart failure associated with RHD had significantly lower systolic (118 vs. $132.4 \mathrm{mmHg}$ ) and diastolic (76.2 vs. $85.7 \mathrm{mmHg}$ ) blood pressures, lower severity of peripheral oedema and a higher mean ejection fraction (48.1\% vs. $38.1 \%)$. The mean eGFR and WBC were significantly higher in patients with RHD-HF. The echocardiographic evaluation is shown in Table 3. Patients with RHD-HF had significantly higher left atrial size (54.6 vs. $46 \mathrm{~mm}$ ), lower LV end systolic diameter and higher ejection fraction and higher mitral inflow variables such as e wave deceleration time. The mean left ventricular endsystolic diameter was lower in RHD-HF patients.

\section{Comparison of oral medications at baseline}

The oral medication use is shown in Table 4. Patients with RHD-HF were significantly more likely to be prescribed beta-blockers (28.9\% vs. $14.4 \%)$, however, the prescription of beta blockers in patients with RHD-HF was not influenced by the presence of atrial fibrillation. At admission $28.3 \%$ of patients with RHD-HF who were in atrial fibrillation received beta blockers versus $29.1 \%$ of those who were not in atrial fibrillation. Patients with RHD-HF were less likely to be prescribed the RAAS blockers (54.3\% vs. $77 \%$ ). The pattern of prescription of other medications was similar in both groups.

\section{Comparison of clinical outcomes}

The clinical outcomes (length of hospital stay, readmission and death) are shown in Table 5. Length of initial stay was available for $863(86.6 \%)$ of the 997 patients; length of stay was considered missing for 42 patients who died inhospital and 92 patients were missing the discharge date. Patients with RHD-HF had a numerically, though nonsignificantly, longer length of initial stay in the hospital (10.5 vs. 8.8 days) but had significantly higher initial hospitalization mortality (9.1\% vs. 3.4\%). Readmission at 60 days was comparable between groups (Figure 2A). Patients with RHD-HF had a slightly higher estimated rate of all-cause death or readmission through 60 days (Figure $2 B$ ), although the difference was not statistically significant. At 180 days, patients with RHD-HF had a similar risk of death (Figure $2 C$ ).

\section{Discussion}

The etiology, medical management, and short-term clinical outcomes of 1,006 Africans with AHF from nine SSA countries were described in the sub-Saharan Africa Survey of Heart Failure (THESUS-HF) (2). In this study, RHD was the third most common cause of heart failure in SSA. RHD has virtually disappeared in high income countries but prevails as a major cause of cardiovascular death and disability in children and young adults in low- 
Table 1 Comparison of sociodemographic and risk factor profile

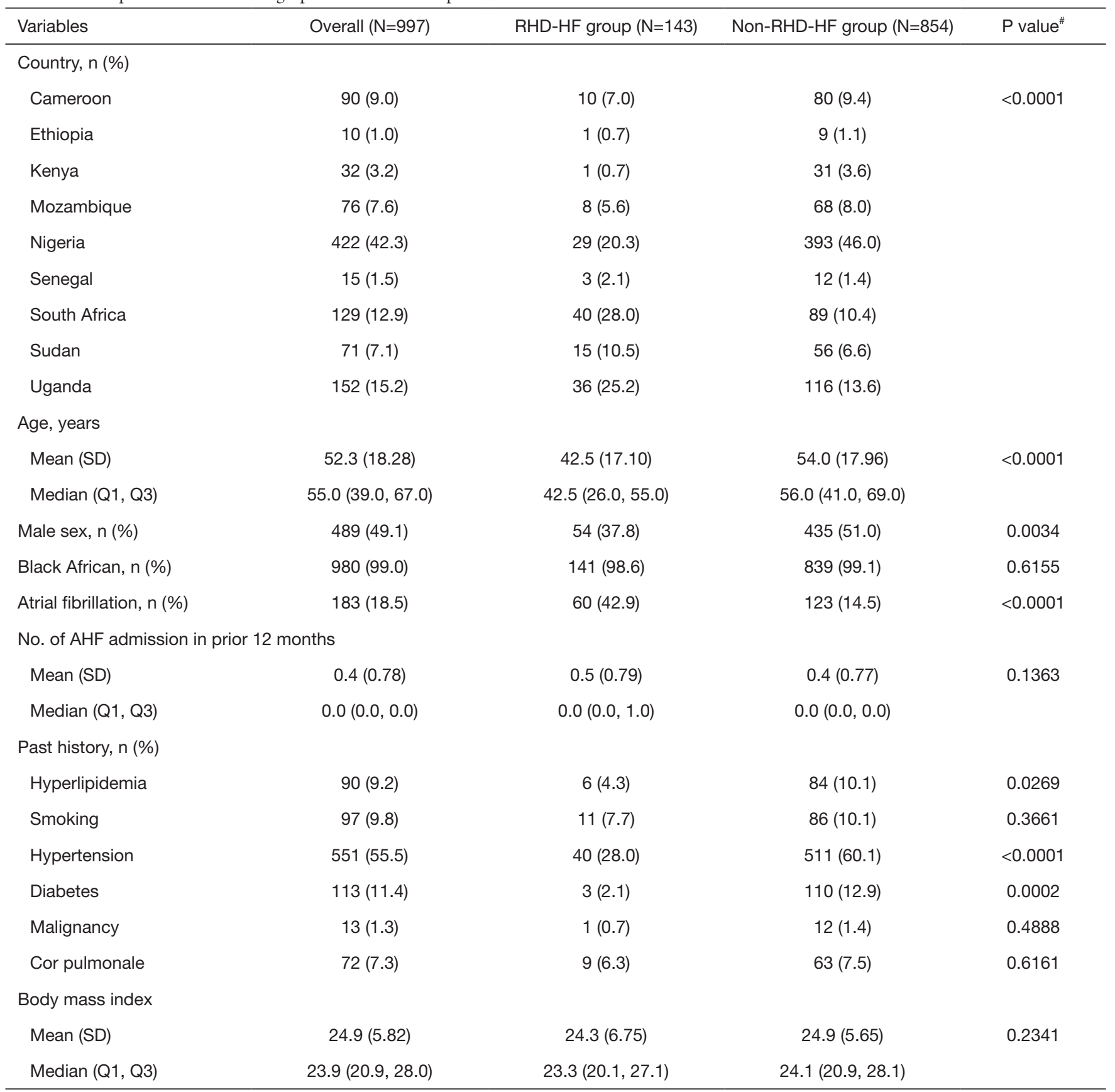

${ }^{\#} \mathrm{P}$ value from $t$-test for continuous variables or from $\chi^{2}$-test for categorical variables. RHD, rheumatic heart disease; SD, standard deviation; Q1, first quartile; Q3, third quartile.

and middle-income countries, including SSA (6-8). The aim of this study was to compare the socio-demographic and risk factor profile, clinical characteristics, and outcomes between patients with RHD heart failure and Non-RHD heart failure in SSA using data from the THESUS heart failure registry (2). These analyses showed that patients with HF associated with RHD were: younger, more were females, were more likely to be in atrial fibrillation, had less hypertension, hyperlipidemia and diabetes, had lower BP, and higher pulse rate and better kidney function but more 
Table 2 Comparison of clinical presentation

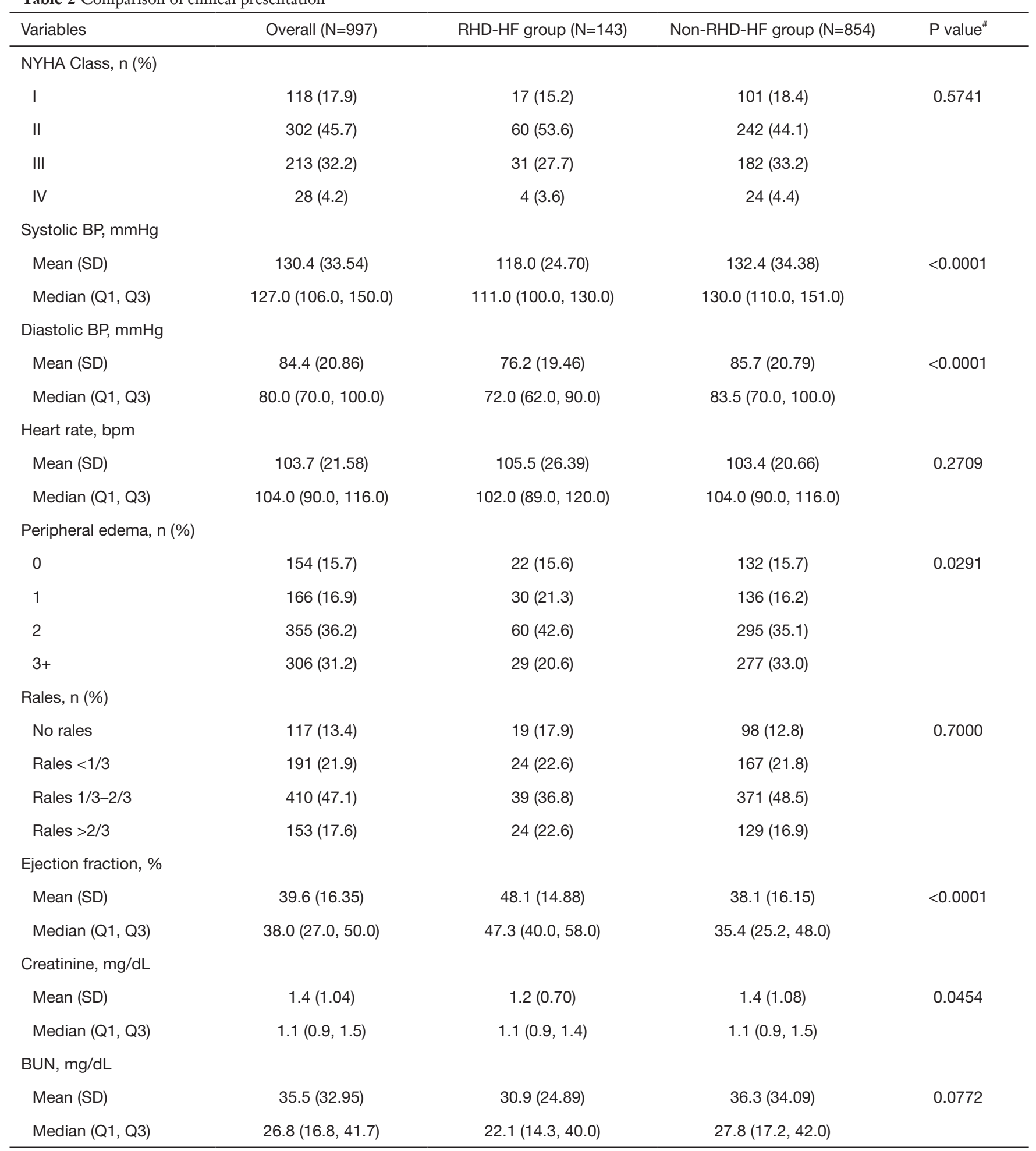

Table 2 (continued) 
Table 2 (continued)

\begin{tabular}{|c|c|c|c|c|}
\hline Variables & Overall $(\mathrm{N}=997)$ & RHD-HF group $(\mathrm{N}=143)$ & Non-RHD-HF group $(\mathrm{N}=854)$ & $P$ value \\
\hline \multicolumn{5}{|c|}{ eGFR, $\mathrm{mL} / \mathrm{min} / 1.73 \mathrm{~m}^{2}$} \\
\hline Mean (SD) & $83.9(47.53)$ & $92.2(59.73)$ & $82.4(44.93)$ & 0.0239 \\
\hline Median (Q1, Q3) & $77.1(55.0,103.8)$ & $79.9(62.1,107.0)$ & $76.8(53.3,103.5)$ & \\
\hline \multicolumn{5}{|l|}{ Cholesterol, mg/dL } \\
\hline Median (Q1, Q3) & $152.1(124.0,187.0)$ & $152.1(114.7,171.6)$ & $153.3(124.8,187.2)$ & \\
\hline \multicolumn{5}{|l|}{ Triglycerides, mg/dL } \\
\hline Mean (SD) & $106.3(53.94)$ & $98.2(40.31)$ & 107.7 (55.92) & 0.1127 \\
\hline Median (Q1, Q3) & $97.9(71.2,124.6)$ & $90.5(67.3,115.7)$ & $97.9(71.2,127.3)$ & \\
\hline Median (Q1, Q3) & $93.7(84.0,117.0)$ & $91.8(82.8,101.7)$ & $95.0(84.6,120.0)$ & \\
\hline \multicolumn{5}{|l|}{ Hemoglobin, g/dL } \\
\hline Mean (SD) & $12.2(2.42)$ & $12.0(2.07)$ & $12.2(2.47)$ & 0.4753 \\
\hline Median (Q1, Q3) & $12.3(10.7,13.7)$ & $12.0(10.5,13.3)$ & $12.3(10.7,13.8)$ & \\
\hline \multicolumn{5}{|l|}{ Lymphocytes, \% } \\
\hline Mean (SD) & $30.3(13.37)$ & $28.4(13.16)$ & 30.6 (13.39) & 0.0986 \\
\hline Median (Q1, Q3) & $30.0(20.0,39.6)$ & $29.0(19.0,36.5)$ & $30.0(20.4,40.0)$ & \\
\hline \multicolumn{5}{|l|}{ Sodium, mmol/L } \\
\hline
\end{tabular}

${ }^{\#} \mathrm{P}$ value from $t$-test for continuous variables, Wilcoxon rank-sum test for ordinal variables, and $\chi^{2}$-test for categorical variables. $\mathrm{RHD}$, rheumatic heart disease; SD, standard deviation; Q1, first quartile; Q3, third quartile; NYHA, New York Heart Association; BUN, blood urea nitrogen; eGFR, estimated glomerular filtration rate; WBC, white blood cell.

leukocytosis. By echocardiography patients with RHDAHD had higher ejection fraction but larger left atria and more diastolic dysfunction. The study also showed that RHD-HF patients were significantly more often prescribed beta blockers, had a significantly worse short-term outcome, especially higher mortality on initial hospitalization. However, the longer-term (60 and 180 days) outcomes did not differ significantly in patients with RHD-HF. Some of the differences observed between patients with RHDAHF and non-RHD AHF may be due to the fact that some specific subgroups of non-RHD AHF are more likely to have specific characteristics, such as higher BP in patients with hypertension related AHF. These differences cannot be explored here in detail due to the limited number of patients presenting with different AHF etiologies.

To our knowledge this is the first study comparing patients with AHF associated with RHD to patients with other etiologies of AHF not related to RHD in SSA. Previous reports from SSA have shown that heart failure affects men and women in the most productive years of their 
Table 3 Comparison of echocardiographic evaluations

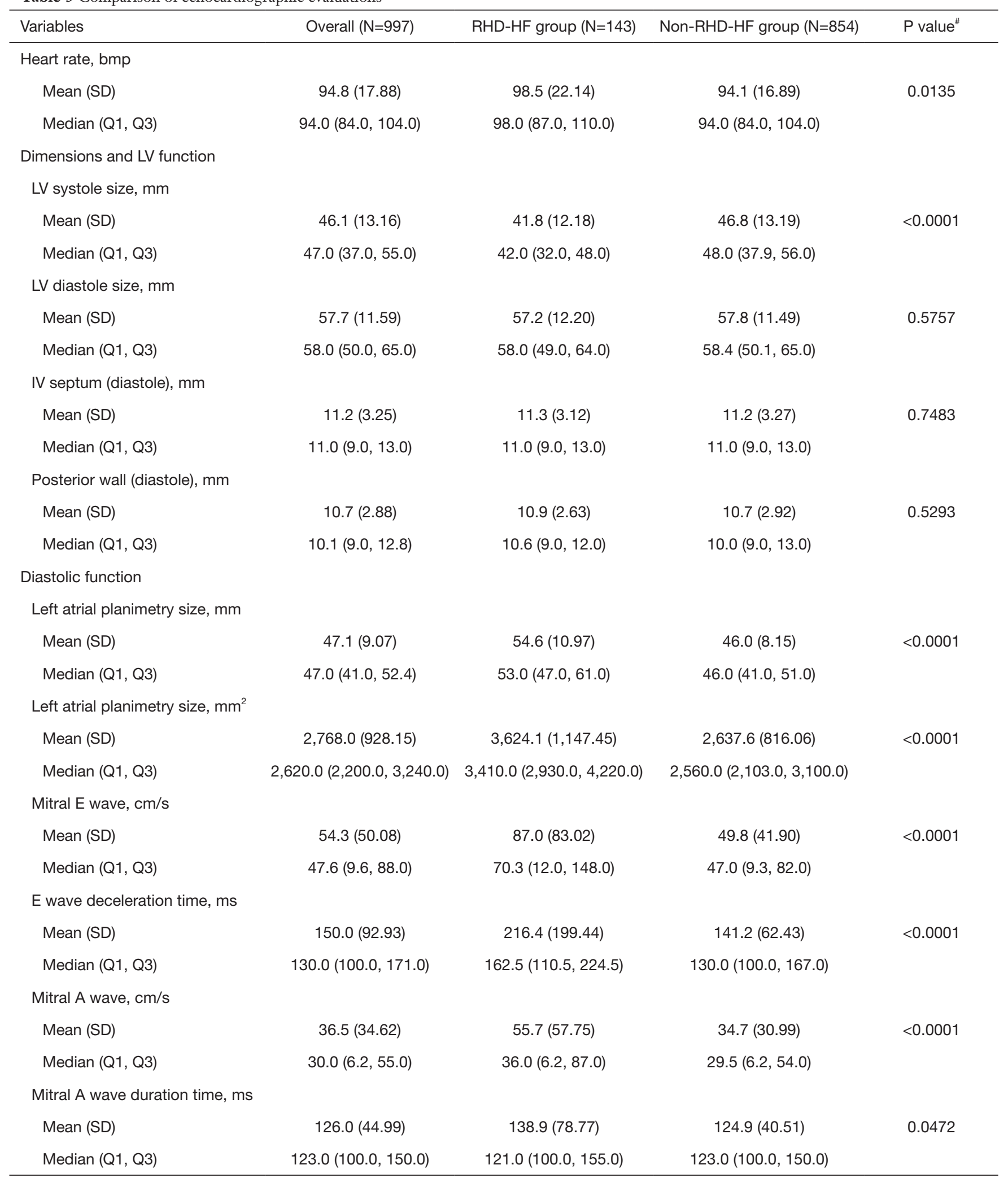

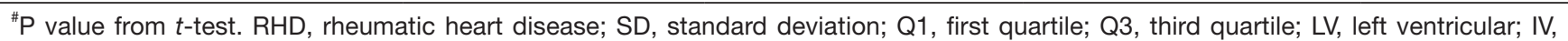
interventricular. 
Table 4 Comparison of prescribed oral medication use at admission

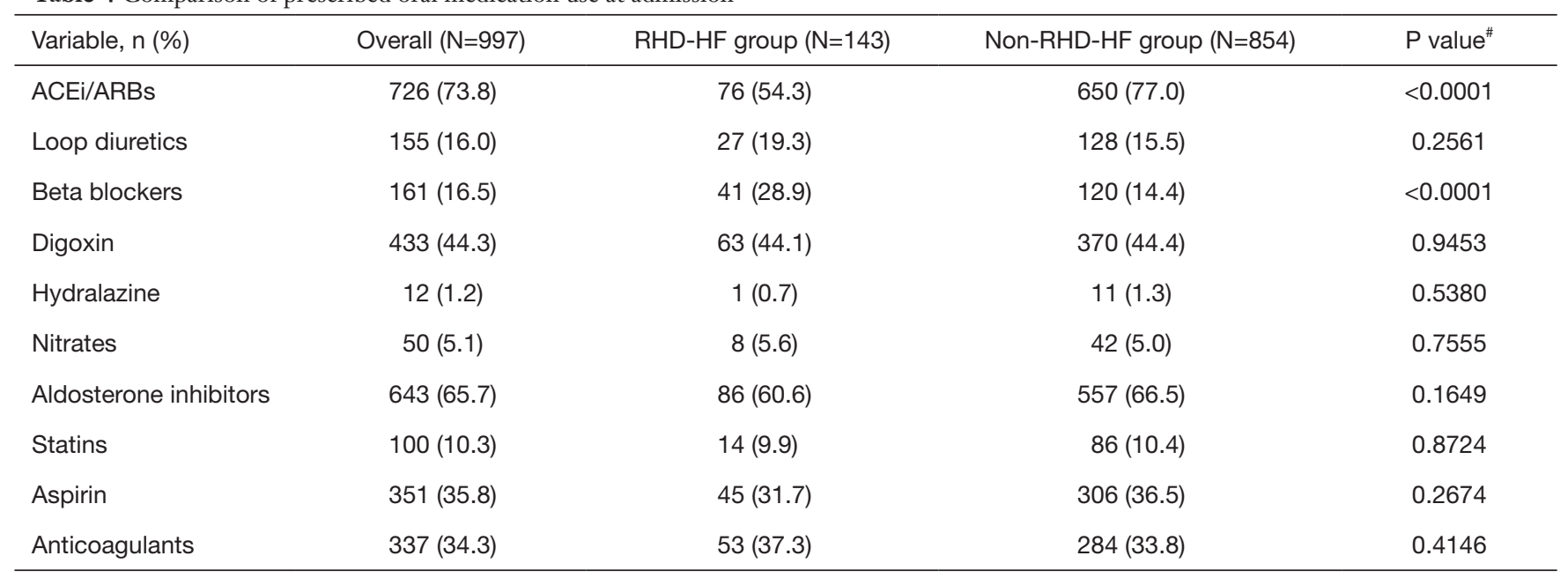

${ }^{\#} \mathrm{P}$ value from $\chi^{2}$-test. RHD, rheumatic heart disease; ACEi, angiotensin converting enzyme inhibitor; ARB, angiotensin II receptor blocker.

Table 5 Comparison of clinical outcomes

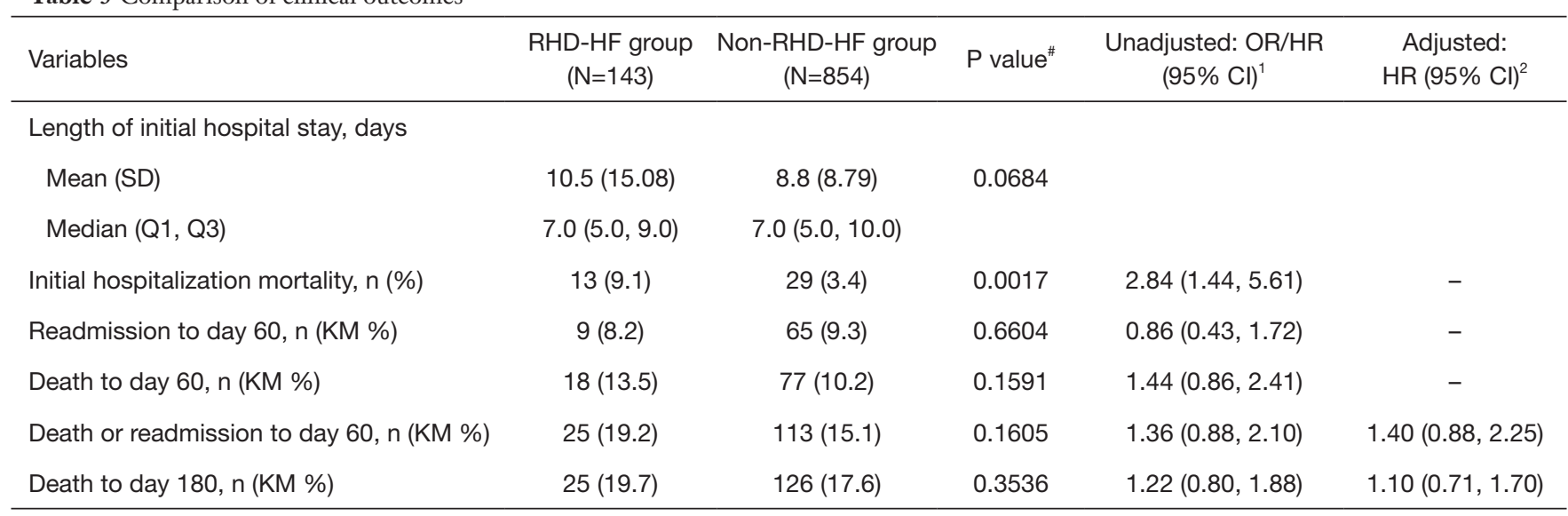

${ }^{\#} \mathrm{P}$ value from ANOVA (F-test) for length of initial stay, log-rank test for time-to event outcomes, and Wald- $\chi^{2}$-from a logistic regression model for in-hospital mortality. ${ }^{1}$ Odds ratio $(95 \% \mathrm{Cl})$ provided for in-hospital mortality outcome. ${ }^{2}$ Multiple imputations used adjusting for the following predictors. Death to day 180: gender, history of malignancy, history of cor pulmonale, history of HIV, smoking status, and baseline values for systolic BP, creatinine, hemoglobin, heart rate, orthopnea, rales, and edema. Death or readmission to day 60: history of hyperlipidemia, history of malignancy, history of cor pulmonale, and baseline values for rales, ejection fraction, BUN, and systolic BP. RHD, rheumatic heart disease; SD, standard deviation; Q1, first quartile; Q3, third quartile; KM, Kaplan-Meier; OR, odds ratio; HR, hazard ratio.

lives with a mean age of 52 years (2). The characteristics and outcomes of AHF related to RHD are not commonly reported and this series addresses a significant knowledge gap addressing RHD associated AHF. In this analysis, patients with RHD-AHF were significantly younger with a mean age of 42 years, compared to patients with other heart failure etiologies. The mean admission blood pressure was lower in patients with RHD-AHF probably because hypertension was the main etiology of non RHD-AHF, increasing this way the blood pressure in this group.

The prevalence of atrial fibrillation among patients with RHD-AHF was $42 \%$ compared to $14.5 \%$ in non RHDAHF. Atrial fibrillation is one of the main complications of RHD and its prevalence increases with severity of valvular lesion. A study in Ethiopia reported a high prevalence of atrial fibrillation in patients with RHD (46.8\%) which is comparable to that reported in this study (9). This was however higher than that reported in the REMEDY study 
A

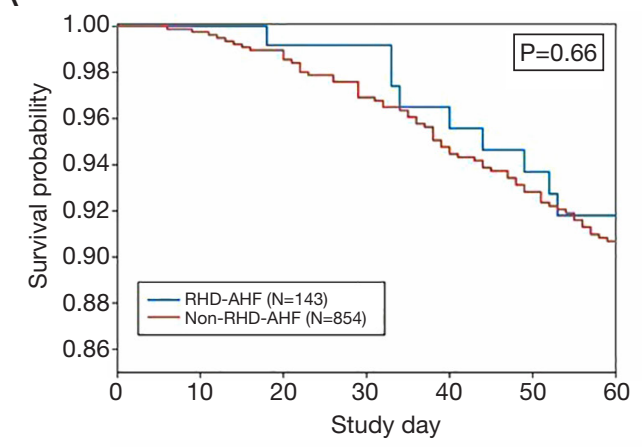

C

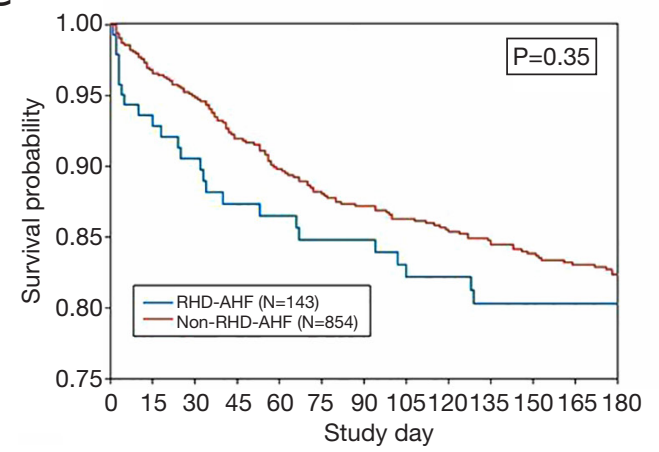

B

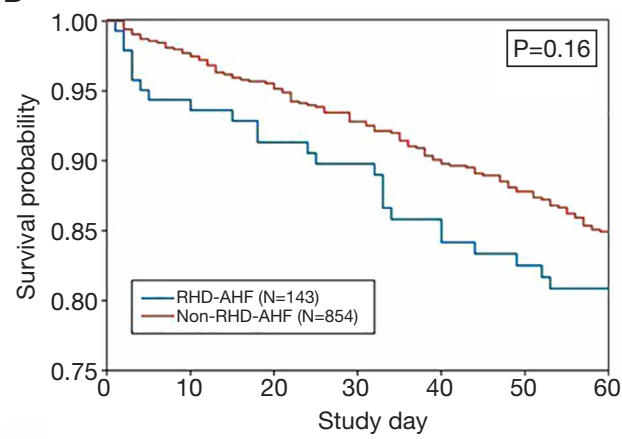

Figure 2 Kaplan-Meier survival plots for (A) all-cause readmission through day 60, (B) all-cause death or readmission through day 60, and (C) all-cause death through day 180 .

with a prevalence of atrial fibrillation of $21 \%$ (10). RHD is associated with a large atrium. An enlarged atrium is predictor of atrial fibrillation as shown by several studies $(11,12)$, a finding that was also observed in the current analysis where patients had larger left atria. These findings suggest that atrial fibrillation is more common in patients with RHD admitted due to AHF then those with chronic, more compensated RHD.

The prescription of beta blockers in patients with heart failure in SSA is low $(1,13)$. In this report, patients with RHD-AHF were more likely to be prescribed beta blockers. This could be explained by a higher prevalence of atrial fibrillation with higher heart rates in these patients. Mitral stenosis, being an indisputable indication for beta blockers could also be a reason for this difference. Patients with RHD-AHF were also less likely to be prescribed angiotensin converting enzyme inhibitors which could be related to the higher left ventricular ejection fraction.

Heart failure is a leading cause of morbidity and hospital readmission (14). In the current analysis, patients with RHD-AHF had higher initial hospitalization mortality, and a trend toward higher initial hospital length of stay.
However, by 6 months follow up the outcomes of patients with AHF were similar regardless of whether the AHF was due to RHD or other causes. This information is addressing an important knowledge gap regarding the course and short-term outcomes of RHD related AHF.

Although we don't know the number of patients with RHD-AHF that underwent surgery, limited access to surgical treatment can explain in part the higher risk of early adverse outcome in patients with RHD-AHF in this analysis. RHD is usually diagnosed late in Africa with patients presenting late with advanced disease leading to high morbidity and mortality (15). The REMEDY study demonstrated that patients with RHD often present with moderate-to-severe multivalvular disease $(63.9 \%)$, heart failure $(33.4 \%)$, and pulmonary hypertension (28.8\%); atrial fibrillation (21.8\%), stroke $(7.1 \%)$, and infective endocarditis (4\%) are also seen (10). In this background of low- and middle-income countries, prohibitive costs and limited access to surgical and catheter-based interventions leave few options for treatment of such late presentations (16). These factors can explain the increased mortality and readmission in patients with heart failure associated with RHD. 


\section{Conclusions}

This series is the first to report on the characteristics and short-term outcomes of patients with RHD related AHF in recent years. Our data suggest that patients with AHF related to RHD are younger, have a higher prevalence of atrial fibrillation and have a worse outcome during the initial admission compared to heart failure related to other etiologies in SSA, however by 6 months their outcomes are similar to other patients with AHF not related to RHD.

\section{Limitations}

The current analysis of the THESUS-HF registry is posthoc. As such it is limited by the nature of the post-Hoc analyses. Some of the comparisons described between RHD-AHF patients and non-RHD-AHF patients may be driven due to differences in specific subgroups of nonRHD-AHF such as hypertensive, HIV or postpartum cardiomyopathy. More detailed analysis of such contrast could not be performed due to the size of the subgroups and hence small numbers of events in each group. Data on the number of patients who underwent surgery for RHD are not available in THESUS-HF. Data regarding the type of valvular lesions in patients with RHD-HF is also unavailable in this cohort. Despite these limitations, this paper serves to highlight an important facet of a neglected disease from the regions where it has the greatest impact in terms of mortality and morbidity. Thus, there is need for increased advocacy on RHD in SSA.

\section{Acknowledgments}

The authors thank all the investigators who participated in the registry.

Funding: None.

\section{Footnote}

Reporting Checklist: The authors have completed the STROBE reporting checklist. Available at https://dx.doi. org/10.21037/cdt-21-112

Data Sharing Statement: Available at https://dx.doi. org/10.21037/cdt-21-112

Conflicts of Interest: All authors have completed the ICMJE uniform disclosure form (available at https://dx.doi. org/10.21037/cdt-21-112). BD, CE, and GC, report grants from Abbott Laboratories, Amgen Inc., Celyad, Cirius Therapeutics Inc., Sanofi, Roche Diagnostics Inc., Trevena Inc., Ventrix, and WindtreeTherapeutics, Inc. The other authors have no conflicts of interest to declare.

Ethical Statement: The authors are accountable for all aspects of the work in ensuring that questions related to the accuracy or integrity of any part of the work are appropriately investigated and resolved. Written informed consent was obtained from each subject who was enrolled into the study. Ethical approval was obtained from the ethical review board of the participating institutions, and the study conformed to the principles outlined in the Declaration of Helsinki (as revised in 2013).

Open Access Statement: This is an Open Access article distributed in accordance with the Creative Commons Attribution-NonCommercial-NoDerivs 4.0 International License (CC BY-NC-ND 4.0), which permits the noncommercial replication and distribution of the article with the strict proviso that no changes or edits are made and the original work is properly cited (including links to both the formal publication through the relevant DOI and the license). See: https://creativecommons.org/licenses/by-nc-nd/4.0/.

\section{References}

1. Agbor VN, Essouma M, Ntusi NAB, et al. Heart failure in sub-Saharan Africa: A contemporaneous systematic review and meta-analysis. Int J Cardiol 2018;257:207-15.

2. Damasceno A, Mayosi BM, Sani M, et al. The causes, treatment, and outcome of acute heart failure in 1006 Africans from 9 countries. Arch Intern Med 2012;172:1386-94.

3. Mocumbi AO. The challenges of cardiac surgery for African children. Cardiovasc J Afr 2012;23:165-7.

4. Zühlke L, Mirabel M, Marijon E. Congenital heart disease and rheumatic heart disease in Africa: recent advances and current priorities. Heart 2013;99:1554-61.

5. Sliwa K, Davison BA, Mayosi BM, et al. Readmission and death after an acute heart failure event: predictors and outcomes in sub-Saharan Africa: results from the THESUS-HF registry. Eur Heart J 2013;34:3151-9.

6. Watkins DA, Johnson CO, Colquhoun SM, et al. Global, Regional, and National Burden of Rheumatic Heart Disease, 1990-2015. N Engl J Med 2017;377:713-22.

7. GBD 2013 Mortality and Causes of Death Collaborators. 
Global, regional, and national age-sex specific all-cause and cause-specific mortality for 240 causes of death, 19902013: a systematic analysis for the Global Burden of Disease Study 2013. Lancet 2015;385:117-71.

8. Global Burden of Disease Study 2013 Collaborators. Global, regional, and national incidence, prevalence, and years lived with disability for 301 acute and chronic diseases and injuries in 188 countries, 1990-2013: a systematic analysis for the Global Burden of Disease Study 2013. Lancet 2015;386:743-800.

9. Yadeta D, Semeredin N, Mekonnen GE. Prevalence and Predictors of Atrial Fibrillation and its Embolic Complications in Patients with Rheumatic Heart Disease at Tikur Anbessa Specialized Hospital, Addis Ababa, Ethiopia. J Health Dev 2019;33:12-6.

10. Zühlke L, Engel ME, Karthikeyan G, et al. Characteristics, complications, and gaps in evidence-based interventions in rheumatic heart disease: the Global Rheumatic Heart Disease Registry (the REMEDY study). Eur Heart J

Cite this article as: Nkoke C, Damasceno A, Edwards C, Davison B, Cotter G, Sani M, Gaeta L, Ogah OS, Mondo C, Ojji D, Suliman A, Yonga G, Ba SA, Dzudie A, Sliwa K. Differences in socio-demographic and risk factor profile, clinical presentation, and outcomes between patients with and without RHD heart failure in Sub-Saharan Africa: results from the THESUS-HF registry. Cardiovasc Diagn Ther 2021;11(4):980990. doi: $10.21037 / \mathrm{cdt}-21-112$ 2015;36:1115-22a.

11. Vaziri SM, Larson MG, Benjamin EJ, et al. Echocardiographic predictors of nonrheumatic atrial fibrillation. The Framingham Heart Study. Circulation 1994;89:724-30.

12. Psaty BM, Manolio TA, Kuller LH, et al. Incidence of and risk factors for atrial fibrillation in older adults. Circulation 1997;96:2455-61.

13. Callender T, Woodward M, Roth G, et al. Heart failure care in low- and middle-income countries: a systematic review and meta-analysis. PLoS Med 2014;11:e1001699.

14. Ross JS, Chen J, Lin Z, et al. Recent national trends in readmission rates after heart failure hospitalization. Circ Heart Fail 2010;3:97-103.

15. Mocumbi AO. Rheumatic heart disease in Africa: is there a role for genetic studies? Cardiovasc J Afr 2015;26:S21-6.

16. Godown J, Beaton A. Handheld echocardiography: a new tool for rheumatic heart disease screening in the developing world? Transl Pediatr 2015;4:252-3. 